\title{
A Prospective Study of Doppler Velocimetry in Pregnancy-induced Hypertension in a Rural Population of a Developing Country
}

\author{
Samir Kumar Hazra, Kamal Kumar Dash', Arunima Chaudhuri², Mrinal Kanti Ghosh ${ }^{3}$, Debdut Banerjee, Sarmistha Guha \\ Departments of Gynecology and Obstetrics, ${ }^{2}$ Physiology and ${ }^{3}$ Radiology, Burdwan Medical College and Hospital, Burdwan, ${ }^{1}$ Department of \\ Gynecology and Obstetrics, North Bengal Medical College and Hospital, West Bengal, India
}

\section{A B S T R A C T}

Background: Pregnancy-induced hypertension (PIH) remains a great challenge to obstetricians. Doppler velocimetry can detect fetal compromise much before other antepartum tests. Aim: The aim of this study is to detect the changes of uterine artery, umbilical artery and middle cerebral artery in PIH by Doppler velocimetry. Subjects and Methods: This prospective study was conducted on hundred subjects with PIH. Doppler studies were carried, and parameters recorded in uterine, umbilical and middle cerebral artery (MCA) were Systolic/Diastolic ratio, Resistance Index, Cerebro-Placental Index (CPI). Fetal outcomes were monitored. Statistical analysis was performed using Epi Info ${ }^{\mathrm{TM}}$ software (Version 3.5.1, CDC, Atlanta). Test for significance was done with student's t-test and Chi-square where applicable. A P-value of $<0.05$ was considered as significant. Results: Among the 100 subjects, 76 (76\%) of fetuses had abnormal and $24 \%$ normal umbilical artery Doppler velocimetry; $62 \%$ had abnormal and $38 \%$ normal MCA Doppler velocimetry; $64 \%$ fetuses had abnormal and $36 \%$ normal CPI. In $95 \%$ of subjects having abnormal umbilical Doppler studies, caesarean section had to be done for acute fetal distress. Incidence of caesarean section was $61 \%$ in abnormal MCA group and $63 \%$ in abnormal CPI group. Among 14 patients who had abnormal uterine artery Doppler, four developed pre-eclampsia, 2 IUGR. In patients with notches in uterine artery Doppler, 38\% developed pre-eclampsia, 38\% had IUGR, 13\% babies were still born and $25 \%$ of newborns required NICU admission. In umbilical artery Doppler, when S/D ratio was abnormal, $60 \%$ developed pre-eclampsia, $40 \%$ had IUGR and $40 \%$ of newborns had to be admitted in NICU. Conclusion: Doppler study for fetal surveillance in pregnancy-induced hypertension is a very useful device and abnormal umbilical artery and uterine artery velocimetry seems to have worse pregnancy outcomes in the present study. Notch as a single parameter is the best indicator with highest sensitivity and positive predicative values. However, combination of parameters is the best indicator.

KEY WORDS: Doppler study, fetomaternal outcome, pregnancy-induced hypertension

\section{INTRODUCTION}

Pregnancy-induced hypertension $(\mathrm{PIH})$ remains a great challenge to obstetricians. Impaired utero-placental blood flow in PIH may result in intrauterine growth restriction (IUGR), placental abruption and intrauterine fetal death (IUFD). ${ }^{[1-3]}$ Doppler velocimetry of uterine artery, umbilical artery and middle cerebral artery can detect fetal defects much before any other antepartum test.

The blood flow characteristics can be quantified by various Doppler indices like the systolic/diastolic ratio $(\mathrm{S} / \mathrm{D}$ ratio $=$ Peak systolic velocity/End diastolic velocity $)$,

\begin{tabular}{|l|l|}
\hline \multicolumn{2}{|c|}{ Access this article online } \\
\hline Quick Response Code & Website: \\
\hline & www.jbcrs.org \\
\cline { 2 - 2 } & \\
\hline
\end{tabular}

resistance index $(\mathrm{RI}=$ Peak systolic velocity- End diastolic velocity/Peak systolic velocity), Cerebro-placental index $(\mathrm{CPI}=$ Middle cerebral artery resistance/umbilical artery resistance) and pulsatility index $(\mathrm{PI}=$ Peak systolic velocity - End diastolic velocity/Mean velocity). ${ }^{[2-5]}$

Forty percent of women with bilateral notches of uterine arteries and high pulsatility index have chances of developing pre-eclampsia, while in $45 \%$, there is chance of developing IUGR. ${ }^{[2-4]}$ Color Doppler is an excellent tool for non-invasive hemodynamic monitoring of PIH patients. It helps to identify the fetuses at risk and predict perinatal morbidity and mortality. ${ }^{[2-6]}$

The present study was conducted in a tertiary care hospital catering a rural population in a developing country to find

\footnotetext{
Address for correspondence Dr. Arunima Chaudhuri, Krishnasayar South, Borehat, Burdwan - 713 102, West Bengal, India. E-mail: arunimachaudhuri4u@gmail.com
} 
the incidence of PIH and detect the changes by Doppler velocimetry of uterine artery, umbilical artery, and middle cerebral artery. Early detection of abnormalities by Doppler may improve perinatal outcome and decrease fetal and maternal morbidity and mortality. The majority of adverse perinatal outcomes in developing countries are placental-associated diseases. Doppler evaluation predicts most occurrences of early-onset preeclampsia and intrauterine growth restriction, and its use in these pregnancies improves perinatal outcomes.

\section{SUBJECTS AND METHODS}

This prospective study was conducted in a time span of one year on hundred subjects in a teaching hospital in West Bengal, catering mainly a rural population of a developing country. Clearance of the Institutional ethical committee and consent of the patients were taken. The study was conducted in the department of Gynecology and Obstetrics in collaboration with the Department of Radiology and Physiology. Inclusion criteria: All antenatal patients with blood pressure $\geq 140 / 90$ after 20 weeks of gestation, with proteinuria $300 \mathrm{mg} / 24 \mathrm{~h}$ or edema or both. Exclusion criteria: Women with multiple pregnancies, chronic hypertension, cephalopelvic disproportion being the cause of previous caesarean section done, having fetus with congenital abnormality, history of antepartum hemorrhage, smoking, hepatic, renal, cardiac disease were excluded. During antenatal checkup after careful history taking, physical examination, blood tests (total count, differential count, erythrocyte sedimentation rate, hemoglobin percentage, bleeding time, clotting time, platelet count, random blood sugar, urea, creatinine, uric acid, VDRL tests, Hepatitis B surface antigen, HIV), Doppler studies were carried out. During Doppler studies parameters recorded were S/D ratio, RI in uterine, umbilical and middle cerebral artery (MCA), Pulsatility Index $(\mathrm{PI}=$ difference between the peak systolic and end-diastolic shift divided by the average shift (A) over the cardiac cycle. The flow velocity waveforms were considered abnormal if there existed an early diastolic notch in uterine artery (in either right or left uterine arteries) and $\mathrm{S} / \mathrm{D}$, RI exceeded $95^{\text {th }}$ percentile of the range of reference; in umbilical artery if S/D, RI exceeded $95^{\text {th }}$ percentile and if end-diastolic flow velocity was absent or reversed. S/D ratio of 3 was considered abnormal after $30^{\text {th }}$ weeks of pregnancy. $\mathrm{CPI} \leq 1$ was considered abnormal.

Outcome measures recorded were: IUGR, IUFD, LBW (low birth weight), Gestational age at birth, Apgar score and Neonatal intensive care unit (NICU) admissions required at birth. In cases with meconium staining of liquor, caesarean section was done.
Statistical analysis was performed using Epi Info ${ }^{\mathrm{TM}}$ software (Version 3.5.1, CDC, Atlanta). Test for significance was done with student's $t$-test and Chi-square where applicable. $P$ value $<0.05 *$ was considered as significant.

\section{RESULTS}

Among hundred PIH cases, 76\% (76/100) of fetuses had abnormal and $24 \%(24 / 100)$ normal umbilical artery Doppler velocimetry ( 24 had UA S/D ratio $<3$ and in 76 UA S/D ratio was $\geq 3$ ). Significant differences were seen in meconium staining of liquor, birth weight, NICU admission, Apgar score and number of caesarean section done among the two groups.

Sixty two percent $(62 / 100)$ of the patients had abnormal and $38 \%(38 / 100)$ normal MCA Doppler velocimetry. Incidence of caesarean section was significantly increased. Among the 32 fetuses who had MCA S/D ratio $\geq 3,29$ had poor fetal and neonatal outcome and out of 19 fetuses who had MCA S/D ratio $<3$, seven had poor outcome.

Sixty four percent (64/100) of fetuses had abnormal and $36 \%(36 / 100)$ normal CPI. Table 1 shows that fetuses with $\mathrm{CPI} \leq 1$ had significantly lower mean gestational age at birth. Meconium staining of liquor during labor for which caesarean section was done was significantly higher $(P<0.001)$ among these fetuses. Caesarean section for acute fetal distress was ninety five percent in abnormal umbilical Doppler group. Incidence of caesarean section was sixty one percent in abnormal MCA group and sixty three percent among abnormal CPI group.

The incidence of low Apgar score $(<7)$ at 5 minutes and NICU admission rate was significantly higher $(P<0.01)$ among fetuses who had CPI $\leq 1$. This group of fetuses also had significantly longer $(P<0.01)$ NICU stay. Out of 32 fetuses who had CPI values of 1 or less, 31 had poor fetal and neonatal outcome; while among 18 fetuses who had CPI $>1$, in 5 outcome was poor. Among 14 patients who had abnormal uterine artery Doppler, four developed pre-eclampsia, two IUGR. Abnormal CPI value had a sensitivity of $86.1 \%$, specificity of $92.8 \%$, positive predictive

\begin{tabular}{|c|c|c|c|}
\hline \multirow[t]{2}{*}{ Neonatal events } & \multicolumn{2}{|c|}{ Cerebroplacental index } & \multirow[t]{2}{*}{$P$ value } \\
\hline & $\leq 1(n=32)$ & $>1(n=31)$ & \\
\hline Gestational age at delivery (weeks) & $34.7(3.1)$ & $36.7(2.9)$ & 0.33 \\
\hline Birth weight $<2500 \mathrm{~g}(n=40)$ & $31(96.9 \%)$ & $9(50 \%)$ & $<0.001 *$ \\
\hline Meconium staining of liquor $(n=33)$ & $25(78 \%)$ & $8(44 \%)$ & $0.04 *$ \\
\hline Apgar score $<7$ at $5 \min .(n=36)$ & $29(90.6 \%)$ & $7(38.9 \%)$ & $<0.001 *$ \\
\hline Caesarean section $(n=24)$ & $20(62.5 \%)$ & $4(22.2 \%)$ & $<0.01^{*}$ \\
\hline NICU admission $(n=24)$ & $22(68.8 \%)$ & $2(11.1 \%)$ & $<0.001 *$ \\
\hline NICU admission $>7$ & $18(56.3 \%)$ & $2(11.1 \%)$ & $<0.01 *$ \\
\hline Days $(n=20)$ & & & \\
\hline
\end{tabular}


value of $96.9 \%$, and negative predictive value of $72.2 \%$ and diagnostic accuracy of $88 \%$.

Table 2 shows S/D ratio and notch had sensitivity of $60 \%$ and positive predictive value of $33.3 \%$ and $37.5 \%$, respectively. Notch as a single parameter is the best indicator with highest sensitivity and positive predicative values. However, combination of parameters is the best indicator. Table 3 shows S/D ratio as the highest sensitivity and positive predictive value index in umbilical artery. Table 4 shows correlation of uterine artery Doppler with pregnancy outcomes. RI was abnormal in seven cases. Among them 29\% patients developed pre-eclampsia, 14\% developed PIH, 29\% had IUGR, 29\% had IUFD, 14\% babies were still born and $25 \%$ of neonates had to be admitted in NICU. Nine patients had abnormal S/D ratio in uterine artery Doppler. Among them, 33\% developed pre-eclampsia, 33\% had IUGR, 22\% had IUFD, $11 \%$ babies were still born and $22 \%$ of neonates had to be admitted in NICU. Eight patients had early diastolic notch in uterine artery Doppler. Among them 38\% patients developed pre-eclampsia, $38 \%$ had IUGR, $22 \%$ had IUFD, $13 \%$ babies were still born and $25 \%$ of neonates had to be admitted in NICU. Table 5 shows correlation of umbilical artery Doppler with pregnancy outcomes. In umbilical artery Doppler, S/D ratio was abnormal in five cases. Sixty percent patients developed pre-eclampsia, 40\% had IUGR, 20\% had IUFD, $20 \%$ babies were still born and $40 \%$ neonates had to be admitted in NICU. RI was abnormal in eight cases. Twelve percent patients developed pre-eclampsia, 25\% had IUGR, and $12 \%$ neonates had to be admitted in NICU. Diastolic flow was absent in one case. The patients who developed pre-eclampsia, had IUGR and IUFD.

\section{DISCUSSION}

$\mathrm{PIH}$ is a common cause of feto-maternal mortality affecting $10 \%$ of pregnant women and is associated with $22 \%$ perinatal deaths. Doppler studies in high-risk pregnancies are more beneficial in the management of perinatal and neonatal outcomes..$^{[1-7]}$

In the present study, $76 \%$ of fetuses had abnormal and 24\% normal umbilical artery Doppler velocimetry; 62\% had abnormal and 38\% normal MCA Doppler velocimetry; 64\% fetuses had abnormal and 36\% normal CPI. In 95\% of subjects having abnormal umbilical Doppler studies, caesarean section had to be done for acute fetal distress. Incidence of caesarean section was $61 \%$ in abnormal MCA group and $63 \%$ in abnormal CPI group. Liberati et al. ${ }^{[7]}$ showed a mean uterine artery $\mathrm{RI} \geq 0.66$ ( $90^{\text {th }}$ percentile) had better sensitivity than the placental and the non-placental uterine artery. The presence of a diastolic notch in the placental uterine artery increased sensitivity $31.7 \%$ for IUGR and $50.0 \%$ for $\mathrm{PIH}$ ) and positive predictive value of the test. ${ }^{[7]}$ In the present study, S/D ratio and notch had sensitivity of $60 \%$ and positive predictive value of $33.3 \%$ and $37.5 \%$, respectively, and $S / D$ ratio had the highest sensitivity and positive predictive value index in umbilical artery. Sieroszewski et al. ${ }^{[8]}$ observed the sensitivity of the notch in 20-24 week in the uterine artery velocimetry for the prediction of PIH and/or IUGR in the III trimester was 73\%, $68 \%$ and the specificity $97.15 \%$.

In studies of Bhatt et al., ${ }^{[9]} 56 \%$ had abnormal S/D ratio in umbilical artery and/or uterine artery. The percentage of abnormal velocimetry seems to be higher in the present study. Sixty percent of these patients delivered IUGR babies. In patients with absent end diastolic velocity and reversed end diastolic velocity, perinatal mortality was 50\%, and 50\% had IUGR babies. ${ }^{[9]}$ Our study found decreased number of preterm births, obstetric interventions and improved outcome morbidity and mortality from the use of Doppler waveform analysis in early pregnancy. Evidence suggested that many pregnancy disorders originate at conception or in early gestation. A retrospective study was conducted by Frusca et al. ${ }^{[10]}$ on 344 hypertensive pregnant women. Abnormal uterine velocimetry was associated with a worse pregnancy outcome. ${ }^{[10,11]}$ Our study was a prospective study, while

\begin{tabular}{|c|c|c|c|c|c|c|c|c|}
\hline Doppler test & True positive & False negative & False positive & True negative & Sensitivity & Specificity & Positive predictive value & Negative predictive value \\
\hline \multicolumn{9}{|l|}{ Uterine artery } \\
\hline$S / D$ & 3 & 2 & 6 & 89 & 60.0 & 93.7 & 33.3 & $97 \cdot 3$ \\
\hline $\mathrm{RI}$ & 2 & 3 & 6 & 89 & 40.0 & 96.7 & 25.0 & 96.7 \\
\hline Notch & 3 & 2 & 5 & 90 & 60.0 & 97.7 & 37.5 & 97.8 \\
\hline Combined & 4 & 1 & 10 & 85 & 80.0 & 89.4 & 28.6 & 98.8 \\
\hline
\end{tabular}

RI - Resistance index; S/D - Systolic/diastolic ratio

\begin{tabular}{|c|c|c|c|c|c|c|c|c|}
\hline \multicolumn{9}{|c|}{ Table 3: Value of umbilical artery Doppler in predicting pre-eclampsia } \\
\hline Doppler test & True positive & False negative & False positive & True negative & Sensitivity & Specificity & Positive predictive value & Negative predictive value \\
\hline SD ratio & 2 & 3 & 3 & 92 & 40.0 & 96.8 & 40.0 & 96.8 \\
\hline RI & 1 & 4 & 7 & 88 & 25.0 & 92.6 & 12.5 & 95.6 \\
\hline Combined & 2 & 3 & 8 & 87 & 40.0 & 91.5 & 20.0 & 96.6 \\
\hline
\end{tabular}

$\mathrm{RI}$ - Resistance index; S/D - Systolic/diastolic ratio 


\begin{tabular}{lccccc}
\hline \multicolumn{5}{c}{ Table 4: Correlation of uterine artery Doppler with } \\
pregnancy outcome (\%) \\
\hline $\begin{array}{l}\text { Uterine artery } \\
\text { Doppler }\end{array}$ & Preeclampsia \% & IUGR & IUFD \% & $\begin{array}{c}\text { Still } \\
\text { birth }\end{array}$ & $\begin{array}{c}\text { NICU } \\
\text { admission \% }\end{array}$ \\
\hline S/D ratio $(n=9)$ & $33(3 / 9)$ & $33(3 / 9)$ & $22(2 / 9)$ & $11(1 / 9)$ & $22(2 / 9)$ \\
RI $(n=7)$ & $29(2 / 7)$ & $29(2 / 7)$ & $29(2 / 7)$ & $14(1 / 7)$ & $14(1 / 7)$ \\
Early diastolic & $38(3 / 8)$ & $38(3 / 8)$ & $38(3 / 8)$ & $13(1 / 8)$ & $25(2 / 8)$ \\
notch $(n=8)$ & & & & & \\
\hline
\end{tabular}

$\mathrm{RI}$ - Resistance index; S/D - Systolic/diastolic ratio; IUGR - Intrauterine growth restriction; IUFD - Intrauterine fetal death; NICU - Neonatal intensive care unit

\begin{tabular}{lccccc}
\hline \multicolumn{5}{c}{ Table 5: Correlation of umbilical artery Doppler with } \\
pregnancy outcome (\%) \\
\hline $\begin{array}{l}\text { Umbilical artery } \\
\text { Doppler }\end{array}$ & $\begin{array}{l}\text { Preeclampsia \% } \\
\text { IUGR \% }\end{array}$ & IUFD \% & $\begin{array}{c}\text { Still } \\
\text { birth } \%\end{array}$ & $\begin{array}{c}\text { NICU } \\
\text { admission } \%\end{array}$ \\
\hline S/D ratio $(n=5)$ & $60(3 / 5)$ & $40(2 / 5)$ & $2(1 / 5)$ & $20 \%(1 / 5)$ & $40(2 / 5)$ \\
RI $(n=8)$ & $12(1 / 8)$ & $25(2 / 8)$ & 0 & 0 & $12(1 / 8)$ \\
Absent diastolic & $100(1 / 1)$ & $100(1 / 1)$ & $100(1 / 1)$ & 0 & 0 \\
flow $(n=1)$ & & & & & \\
\hline
\end{tabular}

RI - Resistance index; S/D - Systolic/diastolic ratio; IUGR - Intrauterine growth restriction; IUFD - Intrauterine fetal death; NICU - Neonatal intensive care unit

study by Frusca et al. ${ }^{[10]}$ was a retrospective one. Uterine artery sensitivity was $90 \%$ as compared to $40 \%$ sensitivity of combined parameters of umbilical artery in the present study, whic h is comparable to the above study.

Messawa et al. ${ }^{[12]}$ divided their subjects into group A (100) subjected to Doppler velocimetry and group B (100) without Doppler velocimetry. Preterm deliveries, preterm as well as full-term neonatal admissions were more frequent in group A than those in group B, i.e., (39\% vs. $26 \%$ ), (56\% vs. $88 \%$ ) (OR $0.2,95 \% \mathrm{Cl} 0.04-0.7)$, and (30\% vs. $57 \%$ ) (OR $0.3,95 \%$ CI 0.2-0.7), respectively. Similarly preterm and full-term neonatal deaths were rare in group A than those in group B, i.e., (9\% vs. 78\%) (OR 0.1, $95 \% \mathrm{CI} 0.02-0.7)$ and (6\% vs. 29\%) (OR 0.2, 95\% CI 0.03$1.8)$, respectively. Emergency caesarean section rate was rare in the subjects with normal Doppler than those with abnormal Doppler (48\% vs. $100 \%$ ) (OR 0.1, 95\% Cl 0.03-0.4) as well as in group B (48\% vs. $82 \%$ ) (OR $0.2,95 \% \mathrm{Cl} 0.1-0.4)$. These outcomes are comparable to the present study.

Future studies can be conducted using Color Doppler imaging, the most appropriate and the commonly used method for the study of ocular circulation, especially during pregnancy. It enables the visualization and flow measurement of retro bulbar blood vessels. Hemodynamic studies have shown that general arteriolar vasoconstriction, which leads to hypoperfusion of target organs, including the eye area, remains the most significant pathological change in preeclampsia. ${ }^{[13]}$ The cardiovascular reactivity adaptation seen in normal pregnancy is absent in gestational hypertension. ${ }^{[14]}$ Thus it may be said that Doppler study for fetal surveillance in pregnancy-induced hypertension is a very useful device in reduction of perinatal morbidity and mortality by timely intervention. ${ }^{[15-18]}$
Strengths of the study: The present study was conducted in a developing country with high incidence of maternal morbidity and mortality. Early detection of abnormal Doppler studies yielded better results in maternal of fetal outcomes as proper intervention in patient care was adopted.

Limitations of our study: The sample was drawn from one limited geographic area; the results cannot properly be generalized to the national population. Second, because of the cross-sectional design, this study had limited extrapolative value. A randomized comparison of routine versus highly selective Doppler waveform and biophysical profile usage may yield more conclusive results. We did not estimate PIGF. We are planning for a larger project in near future with the population at a large in different rural centers of our hospital in collaboration with the National Rural Heath mission.

\section{CONCLUSION}

Doppler study for fetal surveillance in pregnancy-induced hypertension is a very useful device and abnormal umbilical artery and uterine artery velocimetry also seems to have worse pregnancy outcomes in the present study. Notch as a single parameter is the best indicator with highest sensitivity and positive predicative values. However, combination of parameters is the best indicator.

\section{REFERENCES}

1. Harkness UF, Mari G. Diagnosis and management of intrauterine growth restriction. Clin Perinatol 2004;31;743-64.

2. Low JA. The current status of maternal and fetal velocimetry. Am J Obstet Gynecol 1991;164:1049-63.

3. Kurdi W, Cambell S, Aquilina J, England P, Harrington K. The role of color Doppler imaging of the uterine arteries at 20 weeks gestration in stratifying antenatal care. Ultrasound Obstet Gynecol 1998;12:339-45.

4. Imdad A, Yakoob MY, Siddiqui S, Bhutta ZA. Screening and triage of intrauterine growth restriction (IUGR) in general population and high risk pregnancies: A systematic review with a focus on reduction of IUGR related stillbirths. BMC Public Health 2011;11 Suppl 3:S1.

5. Hoffman C, Galan HL. Assessing the 'at-risk' fetus: Doppler ultrasound. Curr Opin Obstet Gynecol 2009;21:161-6.

6. Cruz-Martinez R, Figueras F. The role of Doppler and placental screening. Best Pract Res Clin Obstet Gynaecol 2009;23:845-55.

7. Liberati M, Rotmensch S, Zannolli P, Perrino S, Celentano C, Tiboni GM, et al. Uterine artery Doppler velocimetry in pregnant women with lateral placentas. J Perinat Med 1997;25:133-8.

8. Sieroszewski P, Guzowski G. Prognostic value of the uterine doppler velocimetry at 20-24 gestation weeks for PIH and IUGR development in pregnancy. Ginekol Pol 2005;76:348-57.

9. Bhatt CJ, Arora J, Shah MS. Role of color doppler in pregnancy induced hypertension (a study of 100 cases). Indian J Radiol Imaging 2003;13:417-20.

10. Frusca T, Soregaroli M, Platto C. Uterine artery velocimetry in patients with gestational hypertension. Obstet Gynecol 2003;102:136-40.

11. Espinoza J, Romero R, Nien JK, Gomez R, Kusanovic JP, Gonçalves LF, et al. Identification of patients at risk for early onset and/or severe 
preeclampsia with the use of uterine artery Doppler velocimetry and placental growth factor. Am J Obstet Gynecol 2007;196:326.e1-13.

12. Messawa M, Ma'ajeni E, Daghistani MH, Ayaz A, Farooq MU. The role of doppler ultrasound in high risk pregnancy: A comparative study. Niger Med J 2012;53:116-20.

13. Matias DS, Costa RF, Matias BS, Cláudio Lemos Correia L. Doppler velocimetry of the orbital vessels in pregnancies complicated by preeclampsia. J Clin Ultrasound 2012;40:576-85.

14. Cankar K, Potocnik N, Strucl M. Alteration of skin laser-Doppler flux response to local cooling in gestational hypertension. Clin Auto Res 2010;20:183-90.

15. Sharma S, Singh S, Gujral U, Oberoi U, Kaur R. Uterine artery notching on color doppler ultrasound and roll over test in prediction of pregnancy induced hypertension. J Obstet Gynaecol India 2011;61:649-51.
16. Al-Azad S, Rupa SA, Khan NA. Doppler prediction of perinatal outcome in pregnancy induced hypertension and intrauterine growth retardation. Mymensingh Med J 2010;19:539-42.

17. Słowakiewicz K, Perenc M, Sieroszewski P. Biochemical prenatal tests and uterine artery Doppler examination in prediction of PIH and IUGR in the third trimester of pregnancy. Ginekol Pol 2010;81:352-7.

18. Shahinaj R, Manoku N, Kroi E, Tasha I. The value of the middle cerebral to umbilical artery Doppler ratio in the prediction of neonatal outcome in patient with preeclampsia and gestational hypertension. J Prenat Med 2010;4:17-21.

How to cite this article: Hazra SK, Dash KK, Chaudhuri A, Ghosh MK, Banerjee D, Guha S. A prospective study of doppler velocimetry in pregnancy-induced hypertension in a rural population of a developing country. J Basic Clin Reprod Sci 2013;2:127-31.

Source of Support: Nil, Conflict of Interest: None declared

\section{"Quick Response Code" link for full text articles}

The journal issue has a unique new feature for reaching to the journal's website without typing a single letter. Each article on its first page has a "Quick Response Code". Using any mobile or other hand-held device with camera and GPRS/other internet source, one can reach to the full text of that particular article on the journal's website. Start a QR-code reading software (see list of free applications from http://tinyurl.com/ yzlh2tc) and point the camera to the QR-code printed in the journal. It will automatically take you to the HTML full text of that article. One can also use a desktop or laptop with web camera for similar functionality. See http://tinyurl.com/2bw7fn3 or http://tinyurl.com/3ysr3me for the free applications. 